\title{
Fire Risk Assessment Using Multiattribute Evaluation
}

JOHN M. WATTS, JR.

Fire Safety Institute

P.O. Box 674, Middlebury, Vermont 05753, USA

\begin{abstract}
The problem of developing an expedient, yet credible approach to fire safety evaluation is addressed. Techniques of multiattribute evaluation from the field of management science offer a promising solution to this difficulty. The use of multiattribute evaluation in fire safety is described including techniques that are unique to this type of problem. Details are given on the identification of attributes, assigning attribute weights and values, and selecting an evaluation model. A five-step process for constructing a multiattribute model of fire safety evaluation is presented. The resulting robust structure can provide a means of coupling "hard" fire safety science with inclusive fire risk assessment.
\end{abstract}

KEYWORDS: Buildings, Decision Analysis, Fire Risk Assessment, Fire Safety Evaluation, Multiattribute Evaluation,

\section{INTRODUCTION}

For many situations where a quantitative fire safety evaluation is desirable, detailed fire risk assessment may not be cost-effective nor appropriate. This could be the case where great sophistication is not required, where widespread use necessitates a standardized procedure. For example, the vast majority of existing buildings are not economically amenable to in-depth fire safety analysis.

When formal optimizing algorithms or simulation models do not exist or are inefficient for the application, or where historical data is insufficient or too nonindicative to use statistical methods, there still may be some sensible things to do. Intuition and experience can often provide good but not necessarily optimal solutions. Procedures that work but do not have a formal underlying theory are called heuristics.

One of the most common and most powerful heuristic decision-making techniques is multiattribute evaluation, an approach supported by a large body of knowledge described in the literature of decision analysis and management science. Multiattribute evaluation is used to 
develop simplitied but robust models of complex systems. Values are assigned to important attributes of the problem based on professional judgement and experience. These values are then operated on by some combination of arithmetic functions to arrive at a single score or index. The result is compared with other assessments or to a standard.

In fire safety, multiattribute evaluations are heuristic processes of modeling and scoring fire hazard and exposure factors to produce a rapid and simple estimate of comparative fire risk. Selected attributes represent both positive and negative fire safety features that have varying degrees of accuracy in their measurement. The process heuristically relates these attributes to produce a quick yet robust evaluation of fire safety.

Multiattribute evaluations have been applied to a variety of hazard and risk assessment projects to reduce costs, set priorities, and facilitate use of technical information. Fire safety applications of multiattribute evaluation are referred to by various names such as risk ranking, index systems, rating schedules, point schemes, and numerical grading. The most widely-used procedures have been reviewed in the literature $[1,2]$. These approaches typically use techniques of multiattribute evaluation in an ad hoc manner.

The purpose of this paper is to define explicitly a structure for Fire Risk Assessment using Multiattribute Evaluation (FRAME). By closely following the tenets and models of the decision and management sciences, the robustness and credibility of multiattribute evaluation can be exploited. Robust fire safety evaluation can provide an important link between the complex scientific principles of theoretical and empirical models, and the less than perfect circumstances of nonlaboratory conditions found in real world applications.

\section{MULTIATTRIBUTE EVALUATION}

A multiattribute evaluation is an aggregation of system attributes into a single index to reflect an ordinal evaluation. Meteorologists, for example, realized that temperature alone does not represent the coldness of a winter day. They created the wind-chill factor from a combination of temperature and wind speed to measure overall cooling effect. Such multiattribute evaluations have been widely used in fire safety.

By nature of the circumstances, fire safety decisions often have to be made under conditions where the data are sparse and uncertain. The technical parameters of fire safety evaluation are complex and involve a network of interacting components, the interactions generally being nonlinear and multidimensional. However, complexity and sparseness of data do not preclude useful and valid approaches. Such circumstances are common in decision making in business and other risk venues. Unless such problems are addressed, developments that could be useful to society may be inhibited.

As implied above, fire safety decisions require more than one attribute to capture all relevant aspects of the consequences. If there are $n$ attributes for a decision problem, $x_{1}, x_{2}, x_{3}, \ldots, x_{n}$, then an evaluation function $E\left(x_{1}, x_{2}, x_{3}, \ldots, x_{n}\right)$ needs to be determined over these measures in order to conduct a performance assessment. It has been shown by Keeny and Raiffa [3] that if tradeoffs among the attributes do not depend on the levels of the remaining attributes then a single measure of the overall outcome of a system is given by 


$$
E\left(x_{1}, \ldots, x_{i}, \ldots, x_{n}\right)=\sum w_{i} R_{i}\left(x_{i}\right)
$$

where the $w_{\mathrm{i}}$ are weighting constants greater than zero and the $R_{\mathrm{i}}\left(x_{\mathrm{i}}\right)$ are normalizing functions of the attributes.

Management science has long dealt with this type of problem. A large body of knowledge exists on the subject of Multiattribute Evaluation, closely related to Multiattribute Decision Analysis, Multicriteria Decision Making, and Multiattribute Utility Theory. Literature describing multiattribute theory, methods, and applications is extensive. Summaries and descriptions of the principal methods are found in Yoon and Hwang [4], and Norris and Marshall [5]. Essential components of multiattribute evaluation are the attributes, their weights and values, and the evaluation model for integration of these numbers.

\section{ATTRIBUTES}

Multiattribute evaluation begins with the generation of a list of attributes that provides a means of evaluating goal achievements. These attributes, also called parameters, elements, factors, variables, etc., identify the ingredients of fire safety.

Fire safety attributes are components of fire risk that are quantitatively determinable by direct or indirect measurement or estimate. They are intended to represent factors that account for an acceptably large portion of the total fire risk. Usually they are not directly measurable. This is especially true for existing buildings where only limited information is readily available. Attributes may be either quantitative or qualitative and both types of attributes are important. Selection of attributes should result in a set that is nonconflicting, coherent, and logical.

Fire safety is a complex system with a large number of factors that may affect it. These factors can range from ignitability of personal clothing to availability of a heliport for evacuation. Computational and cognitive feasibility limit our consideration to only a relatively small number of these variables. Also, it is intuitively appealing to postulate that safety from fire is a Paretian phenomenon in that a relatively small number of attributes account for most of the problem. This is supported by general fire loss figures that suggest a small number of factors are associated with a large proportion of fire deaths. It is necessary then, to identify as attributes some defensible combination of factors that account for an acceptable portion of the fire risk.

\section{USFA Study}

A study of fire safety effectiveness statements conducted for the US Fire Administration focused on logical and reproducible means of identifying key life safety variables [6]. This study included an extensive survey of case histories, research and test data, logic diagrams, codes, fire models, reviews, inspection check lists, insurance rating schedules, and personal experience to identify a list of more than one hundred life safety variables. This large number needed to be reduced to a more appropriately-sized subset. 
Reduction was conducted in two steps. The first pass screened the variables for redundancy, applicability, and whether they were components of a well-usined ire safety system. This reduced the list to sixty-six variables. A second pass involved contingency analysis and functional analysis of each candidate variable to determine its independence and its importance. Table 1 is the list of nineteen key life safety variables or attributes resulting from this study. The attributes are placed into four groupings for convenience and clarity only.

\section{TABI, 1. Key Life Safety Variables [ 6]}

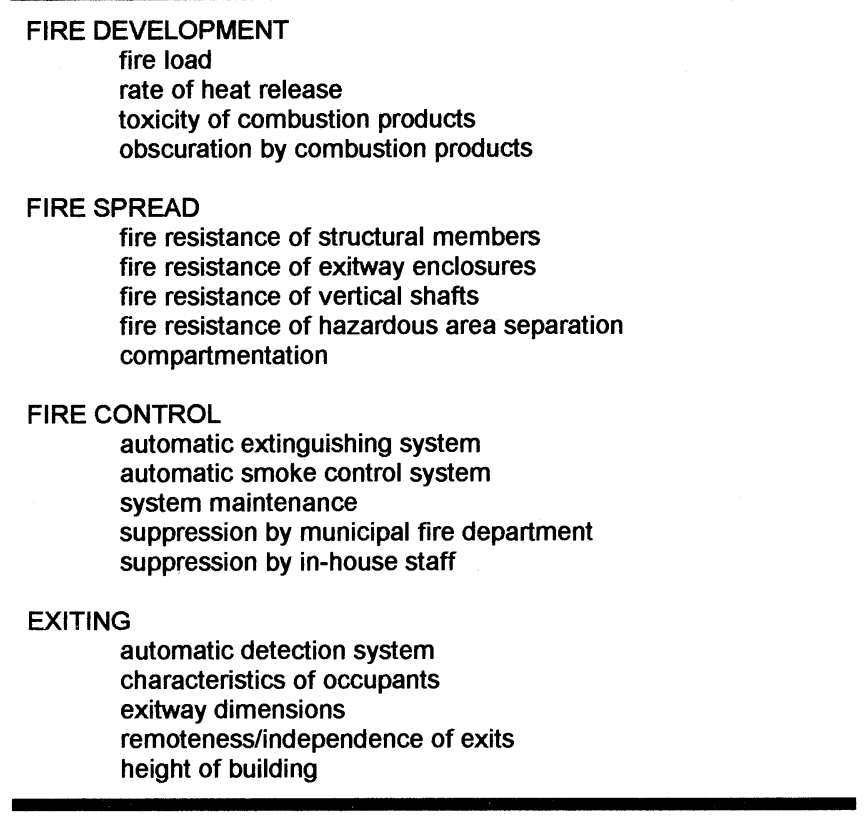

Such a formal process is not typical for most multiattribute evaluations. Selection of attributes is usually more arbitrary, with correspondingly disparate results. Approaches to the selection of attributes generally fall into one of three categories; 1) Delphi, or some less formal consensus process that relies on expert judgement, 2) fire scenarios, ideally based on loss statistics, but usually employing subjective opinion, or 3) cut set of a hierarchical, success tree, providing an inclusive list.

In the final analysis, it is most important that the evaluation vector include only those attributes that vary significantly among buildings and for which the variation is considered meaningful.

\section{ATTRIBUTE WEIGHTS}

Not all fire safety attributes are equally important. The role of weight serves to express the importance of each attribute compared with the others. Hence the assignment of weights is a key component of multiattribute evaluation. 
Although assigning weights by an ordinal scale is usually easier, most multiattribute evaluation methods require cardinal weights. The attribute weights are generaliy normaiized to sum to one, i.e., if $y_{\mathrm{i}}$ is the raw weight of attribute $i$, then

$$
w_{i}=\frac{y_{i}}{\sum^{n} y_{i}} \quad \text { and } \quad \sum_{i=1}^{n} w_{i}=1
$$

This produces a vector of $n$ weights given by $W=\left[w_{1}, \ldots, w_{\mathrm{i}}, \ldots, w_{1}\right]$ where $w_{\mathrm{i}}$ is the resultant weight assigned to the $i$ th attribute.

This relative importance of attributes is defined to be constant across building evaluations. Many weight assessment techniques are used in multiattribute evaluation. To the extent that appropriate data is available, statistical regression is the preferred method of establishing attribute weights. In fire safety this data is often lacking or not indicative of future trends and technology. More subjective hierarchical methods have been found effective.

A hierarchical matrix approach to developing attribute weights was derived in the Edinburgh study of fire safety in health care facilities $[7,8]$. The method uses a hierarchy of decision making levels to generate weights that identify the importance of each fire safety attribute. A particular significance of the method is that the resulting vector is a transparent weighting of attributes with an explicit link to declared fire safety goals and objectives.

The Analytic Hierarchy Process (AHP) is a powerful multiattribute evaluation technique. It has been successfully used to generate attribute ratings as discussed in the next section. However, it may be awkward for establishing attribute weights. For practical purposes the set of attributes used with AHP should be limited to six or seven. Above this number maintaining an acceptable level of consistency in the method is difficult. Most fire safety evaluations deal with fifteen to twenty attributes. Theoretically this constraint can be overcome by partitioning the group of attributes into sets of seven or less. In fire safety evaluation such partitions may compromise independence assumptions or the logic of the hierarchy.

\section{ATTRIBUTE VALUES}

Individual buildings will vary in the degree to which each attribute exists or occurs. Attribute values, also called ratings or grades, are measures of the intensity, level, or degree of danger or security afforded by the attributes in a particular application. To the extent applicable, fire dynamics, fire models, and evacuation simulations can be used to identify ranges of attribute values. However, the selected attributes may be either quantitative or qualitative and methods to make them commensurable are necessary.

Scaling techniques can be used to capture the essential meaning of a qualitative attribute and develop a scale upon which a surrogate measure or value can be based. Quantitative attributes are readily measured or quantified but may require judgement to convert to a compensatory measure. Scaling identifies each individual attribute so that valid and reliable differences among objects can be represented $[9,10]$. 
No scaling model has more intuitive appeal that the Likert scale [10]. Likert scaling rates attributes as $1,2,3,4$, or 5 , reading from unfavorable to favorable. A five-point Likert scale is used predominantly but a more detailed scale such as a seven-point or nine-point scale can also be used if its application does not create undue cognitive stress.

\section{Normalization of Data}

In a typical compensatory evaluation procedure, good performance of one attribute can at least partially compensate for low performance of another attribute. This is also called tradeoff or equivalency. Accommodating tradeoffs of low versus high performance among attributes generally requires normalization of incommensurate data.

Each quantitative attribute typically has a different unit of measurement. Since multiattribute evaluation scoring generally require a homogenous data type, data transformation techniques become necessary. Quantitative attribute values must be normalized to a scale that is common for all attributes. This is accomplished by constructing a normalizing function $R_{\mathrm{i}}\left(x_{\mathrm{i}}\right)$ for each attribute $i$. Normalization aims at obtaining comparable scales that allow interattribute comparison. Consequently, normalized values have dimensionless units and the larger the value becomes, the more preference it has.

Fire safety attributes may be beneficial or detrimental. Beneficial attributes offer monotonically increasing utility, the greater the attribute value, the more its preference, for example fire resistance. Detrimental attributes are monotonically decreasing in utility, the greater the attribute value the less its preference, for example, rate of heat release. Nonmonotonic fire safety attributes are uncommon.

The most common form of normalization is linear. For beneficial attributes the normalized value of attribute $i, r_{\mathrm{i}}$, is given by

$$
r_{i}=\frac{x_{i}-x_{i}}{x_{i}^{\wedge}-x_{i}}
$$

Where $x_{\mathrm{i}}^{\wedge}$ is the ceiling or maximum value of $x_{\mathrm{i}}$, and $x_{\mathrm{i}}^{\vee}$ is the floor or smallest value of $x_{\mathrm{i}}$. Thus, the expression $x_{i}^{\wedge}-x_{i}^{\vee}$ is the range of all values of attribute $x_{i}$. The resultant values have the characteristic that $0 \leq r_{\mathrm{i}} \leq 1$ and the attribute is more favorable as $r_{\mathrm{i}}$ approaches 1 . If, as often happens, $x_{i}^{\vee}=0$, then the normalized value is given by the ratio of the attribute value to the maximum value, $r_{\mathrm{i}}=x_{\mathrm{i}} / x_{\mathbf{i}}^{\wedge}$.

The linear normalized value of a detrimental attribute $i$, is given by

$$
r_{i}=\frac{x_{i}-x_{i}}{x_{i}{ }^{\wedge}-x_{i}}
$$

Again, the resultant values have the characteristic that $0 \leq r_{\mathrm{i}} \leq 1$ and have been adjusted to be consistent with beneficial attributes so that the attribute is more favorable as $r_{\text {: }}$ approaches 1 . 
Where quantitative and qualitative attributes are mixed, the normalized values need to be multiplied by the modulus of the Likert scale used for the qualitative attributes. For example if a five-point Likert scale is used for qualitative data, then the normalized values should be multiplied by five. This is essential to maintain the compensatory capability of the evaluation.

\section{Decision Tables}

Attribute valuation can be simplified by partitioning the attributes into measurable constituent parts. Usually these parts will be directly observable survey items. A survey item is a measurable feature of a building or building space that serves as a constituent part of one or more attributes. Sometimes intermediate subattributes are also used.

Decision tables are common in analysis and documentation for providing orderly representation of information flow in elementary decisions. While many decisions can appear simple by relative comparison, their underlying logic may often be complex. The tabular approach is used to express decision logic in a way that encourages reduction of a problem to its simplest form by arranging and presenting logical alternatives under various conditions.

Decision Tables have been found effective for developing fire safety attribute values from survey items and subattributes [11]. Three properties contribute to their effectiveness in multiattribute evaluation. Decision tables; 1) they provide a disciplined way to assign values to fire safety attributes using data on survey items, 2) they provide a concise and standardized documentation of the detailed design of a multiattribute evaluation, and 3) simplify transition to computerized application.

\section{Analytic Hierarchy Process (AHP)}

Values for fire safety attributes can also be developed using pairwise comparisons and the Analytic Hierarchy Process. AHP has been widely reviewed and applied in the literature and its use is supported by several commercially available user-friendly software packages. Using AHP, the relative importance of each survey item or subattribute is determined by setting up a square matrix, $A$, and making pairwise comparisons.

For each attribute, the set of survey items and subattributes that determine the value of the attribute is identified. Then, each pair of items in a set is examined and a subjective determination made as to which is more important (preferred) and to what extent. So that matrix element $a_{\mathrm{ij}}$ represents how much item $i$ is preferred over item $j$. The degree of preference is assigned from a Likert scale of one to nine. For $n$ items there will be $n(n-1) / 2$ such comparisons. Overall relative importance of each item can then be calculated from the matrix using any of several methods. The best-known and most supported by commercial software is the eigenvalue prioritization method [12].

When using pairwise comparisons and AHP, the number of subattributes or survey items for each attribute should be limited to around seven. This number is congruous with the theory that seven plus or minus two represents the greatest amount of information that an observer can give us about an object from an absolute judgement [13]. 


\section{EVALUATION MODEL}

A multiattribute evaluation may be viewed as a vector of attributes. Transformation of this vector to an appropriate scalar value is the purpose of the evaluation, i.e., formulating an index to represent the effectiveness of the system. In fire safety we do not yet have a thorough understanding of the functional relationships among components so the simple heuristic scoring techrique of additive weighting or scalar product is the most widely used.

An index or score is calculated by adding the contribution from each attribute. Since two items with different measurement units cannot be added, a common numerical scaling system such as normalization, is required to permit addition among attribute values. The total score for each evaluation then can be computed by multiplying the value for each attribute by the importance weight assigned to the attribute and then summing these products over all the attributes. Formally the evaluation score, $\mathrm{S}$, in the additive weight method can be expressed as

$$
S=\sum_{i} w_{i} r_{i}
$$

where $w_{\mathrm{i}}$ is the weight of attribute $i$ and $r_{\mathrm{i}}$ is the normalized value of attribute $i$. Thus, the numeric evaluation is the scalar product of the weighting vector and value vector.

An underlying assumption of additive weighting is that attributes are preferentially independent. Less formal, this means that the contribution of an individual attribute to the total (multiattribute) score is independent of other attribute values. Therefore preferences regarding the value of one attribute are not influenced in any way by the values of the other attributes [14]. Fortunately, studies $[15,16]$ show that additive weighting yields extremely close approximations to "real" value functions even when independence among attributes does not exactly hold.

This model permits very lenient assumptions about individual components of a scale. Nunnally [17] suggests that because each item may contain considerable measurement error or specificity, the importance of this additive model is that it does not take any particular item very seriously. Additive weighting also assumes that the characteristic weights are proportional to the relative value of a unit change in each attribute's value function. This is what makes the method compensatory.

Other evaluation models that have been used in fire safety include weighted product and AHP. In the weighted product model attribute values are multiplied. Weights become exponents associated with each attribute value; a positive value for benefit attributes and a negative power for cost attributes. Formally the evaluation score, $S$, using the weighted product method can be expressed as

$$
S=\prod_{i} x_{i}^{w_{i}}
$$

where $w_{\mathrm{i}}$ is the weight of attribute $i$ and $x_{\mathrm{i}}$ is the value of attribute $i$. 
A weighted product model may produce more variability in results than additive weighting. Since the attribute values are multiplied, a small measurement error in one attribute can generate a significant variation in the score. This makes the model less appropriate when attribute values are the result of subjective determinations with potentially large variances. Weighted product is the evaluation model in the Gretener method used in Europe and Australia $[18,19]$.

The Analytic Hierarchy Process discussed in the previous section on attribute values is also used as a multiattribute evaluation scoring method. It has been adapted to the Edinburgh model for a study of fire safety in dwellings [20]. However, in a fire safety evaluation, this technique has its limitations.

AHP is not as intuitive or transparent as the arithmetic combining of attribute weights and values. Also, as has been previously discussed, it significantly restricts the number of attributes that can be considered. Judgements of pairwise comparisons quickly becomes cognitively onerous as the number of attributes increases. Seven attributes produce twenty-one pairwise comparisons which is approaching the maximum reasonable effort for this process. Even AHP computer software limits the number of attributes to nine. Finally, AHP can be subject to distortion and rank-reversal. However, this does not seem to be a concern if the practical range of the attribute values is known.

\section{SUMMARY}

Fire safety evaluation involves many factors that are hard to assess in a uniform and consistent way. Analysis of such a complex system is difficult but possible as evidenced by activities in the fields of nuclear safety and environmental protection. Detailed fire risk assessment can be an expensive and labor intensive process. Multiattribute evaluations are popular because of their high utility and relative ease of application.

The purpose of a multiattribute evaluation is to provide a useful aid to decision making. Usefulness requires the methodology to be simple yet credible. Applying it must be easy but sophisticated enough to provide a minimum of technical validity. Credibility can also be improved through consistency and transparency. The approach should be systematic and clearly discernible to all interested parties that the relevant technical issues have been appropriately covered.

Specific steps for setting up a fire risk assessment procedure using multiattribute evaluation are outlined below. Buildings with the same set of attributes but differing in attribute values can use the same procedure for evaluation. If a group of buildings has a different set of attributes, then a new procedure to evaluate these buildings can be constructed. Setting up a procedure for fire safety evaluation involves five basic steps.

Step 1. Identify the set of attributes that characterize fire safety in the group of buildings to be evaluated. These attributes must be generated for each application where the important characteristics have significant differences. The number of attributes depends on the nature of the problem. 
Step 2. Develop an importance weighi for each attribute. These weights will remain constant for the specific group of buildings being evaluated.

Step 3. Develop methods for assigning values to each attribute for each building. This is the most challenging step and requires the most documentation for credibility. Likert scaling should be used for qualitative attributes and survey data must be normalized to commensurable units.

Step 4. Select an evaluation model. The additive weighting model is recommended for its simplicity and extensive history of use in fire safety and other decision science applications. Implement the model by collecting data on buildings to be evaiuated, converting this data to attribute values, and applying the evaluation model to calculate the index or score for the building.

Step 5. Validate and calibrate the evaluation procedure. Validity indicates the procedure measures what it was designed to measure. An evaluation procedure cannot be proven valid. Instead, evidence is collected either to support or refute validity. When a sufficient amount of data supporting validity is amassed, the procedure is declared to be valid. If evaluation includes a norm or acceptable level, then the procedure must also be calibrated.

In addition to these procedural steps, criteria have been proposed as an aid in future development and assessment of fire safety evaluation procedures [21]. An example of the application of this structure to fire risk assessment for network integrity in telecommunications facilities is presented elsewhere [22].

Multiattribute evaluation is a simple but effective technique from the field of decision analysis that can deal with the complexity of fire risk assessment. In this approach each building is represented by values on each of several attributes and each value is weighted by the importance of the corresponding attribute. Complexity in evaluation is thus reduced by dealing with one attribute at a time. Complexity in integration of these values is reduced by applying a simple mechanical formula.

While performance-based fire safety design is being developed to address five to ten percent of new buildings, multiattribute evaluation focuses on the other end of the scale of building sophistication. Where it is efficient to assume that the identified parameters adequately address the fire risk, multiattribute evaluation has been found effective. The insurance industry has long used this approach. In the U.S., multiattribute evaluation is widely accepted as an alternative to the Life Safety Code [23] for certain occupancies [24], and for all existing buildings covered by the BOCA National Building Code [25].

Many multiattribute evaluation algorithms can be easily executed on personal computers. The electronic spreadsheet is an especially powerful tool for multiattribute evaluation analysis. It can readily store and manipulate evaluation vectors. A large number of commercial software packages are available for additive weighting and AHP scoring methods. Several computer programs specific to fire safety evaluation have also been developed, including ALARM [26], COFRA [22], FREM [19], and PC-based FSES [27]. 
Multiattribute evaluation produces a meaningful index from multidimensional information to evaluate fire safety. It is a relatively simple model that does not rely exclusively on demonstrated principles of physical or managernent science. Still, its credibility is enhanced to the extent that such principles are employed.

\section{REFERENCES}

1. Watts, J.M., "Fire Risk Ranking", in SFPE Handbook of Fire Protection Engineering, 2nd ed., P. J. DiNenno, ed., pp. 5-12 to 5-26, National Fire Protection Association, Quincy, MA, 1995.

2. Marchant, E.W., "A Cost-effective Approach to Fire Safety, Paper I - Points Scheme", International Fire Security and Safety Conference, 1984.

3. Keeny, R.L. and Raiffa, H. Decisions With Multiple Objectives, John Wiley, New York, 1976.

4. Yoon, K.P. and Hwang, C., Multiple Attribute Decision Making, Sage Publications, London, 1995.

5. Norris, G.A. and Marshall, H.E., "Multiattribute Decision Analysis Method for Evaluating Buildings and Building Systems", NISTIR 5663, National Institute of Standards and Technology, Gaithersburg, MD, 1995.

6. Watts, J.M., Milke, J.A. Bryan, J.L., Dardis, R. and Branigan, V., "A Study of Fire Safety Effectiveness Statements", Department of Fire Protection Engineering, University of Maryland, College Park (NTIS, PB-299169), 1979.

7. Stollard, P., "The Development of a Points Scheme to Assess Fire Safety in Hospitals", Fire Safety Journal, 7:2, 145-153, 1984.

8. Marchant, E.W., "Fire Safety Engineering - A Quantified Analysis", Fire Prevention, 210, 34-38, 1988.

9. Torgerson, W.S., Theory and Methods of Scaling, John Wiley, New York, 1958.

10. McIver, J.P. and Carmines, E.G., Unidimensional Scaling, Sage Publications, London, 1981.

11. Watts, J.M., Budnick, E.K. and Kushler, B.D., "Using Decision Tables to Quantify Fire Risk Parameters", Proceedings - International Conference on Fire Research and Engineering, pp. 241-246, Society of Fire Protection Engineers, Boston, 1995.

12. Saaty, T.L., Multicriteria Decision Making: The Analytic Hierarchy Process, RWS Publications, Pittsburgh, 1990.

13. Miller, G.A., "The Magic Number Seven, Plus or Minus Two", Psychological Review, 63, 81-97, 1956. 
14. Fishburn, P.C., "Utility independence on subsets of product sets", Operations Research, 24, 245-255, 1976.

15. Edwards, W., "Use of multiattribute utility measurement for social decision making", in Conflicting Objectives in Decisions, D.E. Bell, R.L. Keeny, and H. Raiffa, eds., pp. 247-276, John Wiley, New York, 1977.

16. Farmer, T.A., "Testing the robustness of multiattribute utility theory in an applied setting", Decision Sciences, 18, 178-193, 1987.

17. Nunnally, J.C., Psychometric Theory, McGraw-Hill, New York, 1978.

18. Gretener, M., "Fire Risk Evaluation", Association of Cantonal Institutions for Fire Insurance (VKF), Society of Engineers and Architects (SIA), and Fire Prevention Service for Industry and Trade (BVD), Zurich, 1980.

19. Watts, J.M., "Software Review: Fire Risk Evaluation Model", Fire Technology, 31:4, 369-371, 1995.

20. Shields, T.J. and Silcock, G.W. "An Application of the Hierarchical Approach to Fire Safety", Fire Safety Journal, 11:3, 235-242, 1986.

21. Watts, J.M., "Criteria for Fire Risk Ranking", Fire Safety Science - Proceedings of the Third International Symposium, G. Cox and B. Langford, eds., pp 457-466, Elsevier, London, 1991.

22. Budnick, E.K., McKenna, L.A., and Watts, J.M., "Quantifying Fire Risk for Telecommunications Network Integrity", Fire Safety Science - Proceedings of the Fifth International Symposium, Y. Hasemi, ed. (in press).

23. NFPA 101 Life Safety Code, National Fire Protection Association, Quincy MA, 1994.

24. NFPA 101A Guide on Alternative Approaches to Life Safety, National Fire Protection Association, Quincy MA, 1995.

25. "Existing Structures", chapter 34 in The BOCA National Building Code, pp. 319-328, Building Officials and Code Administrators International, Country Club Hills, IL, 1996.

26. Webber, S.F. and Lippiatt, B.C., "Cost-Effective Compliance with Fire Safety Codes", Fire Technology, 32:4, 291-296, 1996.

27. Hughes Associates, Inc., "Fire Safety Evaluation System (FSES) for Business Occupancies Software (ver. 1.0 for Windows $($ ) Users' Manual”, NIST-GCR-96-692, National Institute of Standards and Technology, Gaithersburg, MD, 1996. 